\title{
Nurse-Guided Internet-Delivered Cognitive Behavioral Therapy for Insomnia in General Practice: Results from a Pragmatic Randomized Clinical Trial
}

\author{
Tanja Van der Zweerde ${ }^{a}$ Jaap Lancee ${ }^{b, c}$ Pauline Slottje $^{d} \quad$ Judith E. Bosmans ${ }^{e}$ \\ Eus J.W. Van Someren ${ }^{f, g}$ Annemieke van Straten ${ }^{a}$ \\ a Department of Clinical Psychology, Amsterdam Public Health Research Institute, Vrije Universiteit, Amsterdam, \\ The Netherlands; ${ }^{b}$ Department of Clinical Psychology, University of Amsterdam, Amsterdam, The Netherlands; \\ ${ }^{c}$ PsyQ Amsterdam, Amsterdam, The Netherlands; ${ }^{d}$ Department of General Practice and Elderly Care, Academic \\ Network of General Practice (ANH), Amsterdam Public Health Research Institute, Amsterdam UMC, Vrije Universiteit

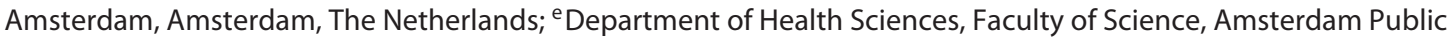 \\ Health Research Institute, Vrije Universiteit Amsterdam, Amsterdam, The Netherlands; ${ }^{f}$ Department of Sleep and \\ Cognition, Netherlands Institute for Neuroscience, an Institute of the Royal Netherlands Academy of Arts and \\ Sciences, Amsterdam, The Netherlands; ${ }^{9}$ Departments of Integrative Neurophysiology and Psychiatry, Centre for \\ Neurogenomics and Cognitive Research, VU University Medical Centre, Amsterdam, The Netherlands
}

\section{Keywords}

Cognitive behavioral therapy, nurse-guided · General practice $\cdot$ Insomnia $\cdot$ Internet interventions $\cdot$ Sleep disorder

\begin{abstract}
Introduction: Guidelines recommend cognitive behavioral therapy for insomnia (CBT-I) as the first line of treatment for insomnia in general practice, but CBT-I is rarely available. Nurse-guided Internet-delivered CBT-I might be a solution to improve access to care. Objective: We aimed to determine the effectiveness of nurse-guided Internet-delivered CBT-I (I-CBT-I) on insomnia severity experienced by patients in general practice. Methods: Nurse-guided I-CBT-I ("i-Sleep") was compared to care-as-usual (and I-CBT-I after 6 months) in 15 participating general practices among 134 patients ( $\geq 18$ years old) with clinical insomnia symptoms. Assessments took place at 8, 26 and 52 weeks. Primary outcome was self-reported insomnia severity (Insomnia Severity Index) at 8 weeks. Secondary outcomes were sleep diary indices, depression and anxiety symptoms (Hospital Anxiety and
\end{abstract}

\begin{tabular}{ll}
\hline karger@karger.com & (C) 2020 The Author(s) \\
www.karger.com/pps & Published by S. Karger AG, Basel Karger \\
& This article is licensed under the Creative Commons Attribution- \\
Karger & NonCommercial-NoDerivatives 4.0 International License (CC BY- \\
NC-ND) (http://www.karger.com/Services/OpenAccessLicense). \\
Usage and distribution for commercial purposes as well as any dis- \\
tribution of modified material requires written permission.
\end{tabular}

Depression Scale), fatigue, daytime consequences of insomnia, sleep medication and adverse events. Results: Two thirds of the 69 intervention patients ( $n=47 ; 68 \%$ ) completed the whole intervention. At the posttest examination, there were large significant effects for insomnia severity (Cohen's $d=1.66$ ), several sleep diary variables (wake after sleep onset, number of awakenings, terminal wakefulness, sleep efficiency, sleep quality) and depression. At 26 weeks there were still significant effects on insomnia severity $(d=1.02)$ and on total sleep time and sleep efficiency. No significant effects were observed for anxiety, fatigue, daily functioning or sleep medication. No adverse events were reported. Conclusions: Nurse-guided I-CBT-I effectively reduces insomnia severity among general practice patients. I-CBT-I enables general practitioners to offer effective insomnia care in accordance with the clinical guidelines.

(c) 2020 The Author(s)

Published by S. Karger AG, Basel

Trial registration: Netherlands Trial register NTR 5202 (April 17, 2015), http://www.trialregister.nl.
Dr. Tanja van der Zweerde

Department of Clinical Psychology, Faculty of Behavior and Movement Sciences VU University, Van der Boechorststraat 7

NL-1081 BT Amsterdam (The Netherlands)

t.vander.zweerde@vu.nl 


\section{Introduction}

With prevalence rates between 7 and $10 \%$ in the general population [1], clinical insomnia is the second most prevalent mental disorder [2]. In general practice, fatigue and sleeplessness are respectively the first and third most reported complaints, and insomnia is estimated to occur in as many as $30 \%$ of general practice patients $[1,3]$. Insomnia leads to a high disease burden on its own but also increases the risks of developing other psychiatric problems $[4,5]$ and cardiovascular diseases [6]. Insomnia, and its comorbid problems, leads to high societal costs, mainly due to productivity losses $[7,8]$.

Cognitive behavioral treatment for insomnia (CBT-I) is an evidence-based treatment with large effects on insomnia severity (demonstrated in several meta-analyses, e.g. Hedges' $g=0.98$ [9]). CBT-I is recommended by (inter)national guidelines [10-13] as the first treatment option for insomnia. In general practice, somewhat smaller effects are reported, but still in the moderate range (Hedges' $g=0.40$ [14]). Despite these moderate effects and the guideline recommendations, sleep medication is still the most commonly used treatment in general practice: $60 \%$ of new insomnia patients generally only receive medication [15]. Even though general practitioners (GPs) acknowledge the importance of CBT-I, they lack the time and/or knowledge to offer it themselves and there is a lack of CBT-I trained therapists to refer to [16]. Internet-delivered CBT-I (I-CBT-I) presents a promising way to increase the availability of CBT-I and to enable GPs to adhere to insomnia guideline recommendations.

Internet treatments usually consist of a number of lessons in which an evidence-based intervention is explained. This might be text-based, video-based or a mixture. Another core feature is that patients receive assignments. They need to apply techniques they read about in the online lessons to their own daily lives. The treatment might be offered without any support, but we know that the adherence and effects are better when it is offered with some type of support [17]. In general, guided Internet treatments seem as effective as face-to-face treatments [18], and this is also demonstrated for I-CBT-I [19, 20]. However, these results are mostly based on self-selected, specifically recruited online samples of help seekers [19, 21]. It is not known whether I-CBT-I also works among patients in a routine general practice. This is what we want to examine in the current study.

In the Netherlands almost all GPs employ a mental health nurse practitioner. Those nurse practitioners provide psychosocial support or treatment for people with mild mental health symptoms. They vary in their background and training but most often they are either mental health nurses or recently graduated psychologists. In this study we refer to them as "nurses." The nurses delivered the I-CBT-I and provided online feedback. We opted for nurse practitioners because not enough trained psychologists are available in the Netherlands. More importantly, in the Dutch health care system, patients who are referred to a psychologist because of insomnia have to pay for treatment out of pocket. The care provided by nurses in general practice falls under the GP care and is fully reimbursed. Therefore, this is more feasible for offering insomnia treatment to patients.

The primary objective of this pragmatic randomized controlled trial was to assess whether nurse-guided ICBT-I is effective in routine general practice compared to care as usual (CAU) on insomnia severity. Secondarily, sleep diary indices, mental health complaints and daily functioning were assessed. Effects were studied immediately after treatment (posttest; 8 weeks), after 26 weeks and (in the intervention group only) after 52 weeks.

\section{Material and Methods}

\section{Research Design}

This 2-arm, pragmatic randomized clinical trial compared ICBT-I to CAU. Patients in the intervention group were also allowed to receive CAU. Patients in the control group received the intervention after the 26-week assessment if they still wanted it. Randomization charts were made for each nurse separately, using random sequence block randomization (blocks of 2, 4 or 6) on a 1:1 ratio and stratified by sleep medication use. Participants were randomized using randomization charts created by an independent researcher. A second independent researcher performed the randomization. The primary researcher (T.Z.) was blinded to treatment allocation. After allocation, the primary researcher, participants, nurses and GPs were informed of the randomization outcome. Participants in CAU were informed they would be offered I-CBT-I after 6 months. GPs strongly requested also being informed when patients were randomized to CAU so they could offer other treatments and patients would not be left on their own. The Medical Ethics Committee of the VU Medical Centre Amsterdam approved this trial (registration No. 2015/258), and it was registered with the Dutch Trial Registry (NTR5202, April 17, 2015). The detailed protocol is available elsewhere [22].

\section{Power}

In the original protocol we calculated our power based on an independent $t$ test. To ensure $80 \%$ statistical power with a 2 -sided $\alpha$-value of 0.05 , assuming a conservative effect size of Cohen's $d=$ 0.50 on the primary outcome insomnia severity [23] and expecting a risk of $20 \%$ dropout [24], we aimed to include 160 participants. 


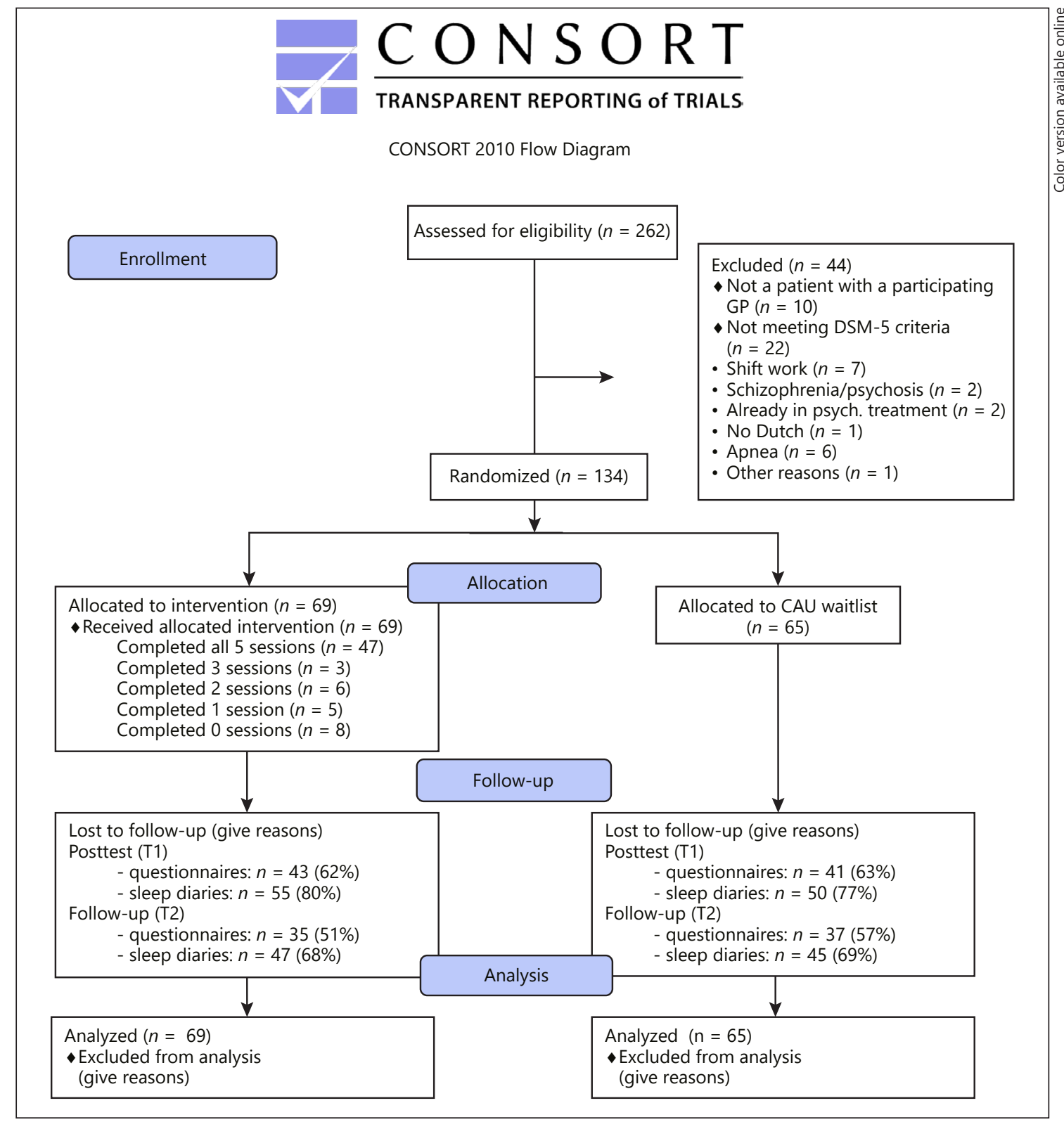

Fig. 1. CONSORT flowchart of participant flow throughout the trial.

When we analyzed the data, we decided it was more appropriate to employ a statistical procedure taking baseline levels into account. Based on this within-between group analysis and the achieved sample size $(n=134)$, we obtained a power of $0.8(\alpha=$ 0.05 ) to detect an effect size of $d=0.24$.

\section{Recruitment}

Recruitment leaflets were distributed in print and on screens in waiting rooms. GPs and nurses were asked to alert all possible eligible insomnia patients who consulted them to the trial. GPs and nurses received laminated summaries of the trial and how to ex- plain it to patients, to use during consultations. Additionally, GPs sent invitation letters and leaflets to patients who had consulted them for insomnia in the past year. All recruitment materials referred patients to our study website which explained the study in more detail. Patients could leave their e-mail address to show that they were interested. They then received an e-mail with a link to a screening questionnaire. Eligible patients received all information in printed form, a sleep diary, informed consent form and stamped return envelope. Patients were randomized after returning the completed sleep diary and informed consent. The inclusion period was extended from originally 18 months to 30 months. We ended 
inclusion in April 2018, at 134 of the planned 160 participants for pragmatic reasons (Fig. 1 for a flow chart). ${ }^{1}$

\section{Eligibility Criteria}

Inclusion criteria were: (1) 18 years or older; (2) reporting lying awake for $\geq 30$ min a night for $\geq 3$ nights per week for $\geq 3$ months; and (3) causing clinically significant distress or impairment in daily functioning. The duration and frequency of insomnia complaints were assessed in an online screening questionnaire. The consequences during the day were assessed with the Daytime Consequences items [25]. This scale assesses 6 domains of functioning: energy, relationships, mood, concentration, productivity and sleepiness. Each domain was measured using a 5-point Likert scale ( $0=$ not disturbed, $4=$ very disturbed $)$. We defined daytime consequence as scoring at least a 3 on one of the domains. These inclusion criteria (sleep complaints and consequences for daily functioning) were chosen because they resemble the DSM- 5 criteria for an insomnia disorder [26]. Exclusion criteria were: (1) no access to the Internet; (2) inadequate proficiency in Dutch; (3) meeting sleep apnea criteria; (4) pregnancy or breastfeeding; (5) working night shifts; (6) other current psychological treatment; (7) suicidal ideation or (8) schizophrenia or psychotic disorder. In order to identify sleep apnea, we used the Screening for Sleeping Disorders questionnaire [27]. Patients with a positive score for suspected untreated sleep apnea (reporting snoring, breathing stops and excessive daytime sleepiness) were excluded. Suicidal ideation over the past month was assessed using 5 MINI diagnostic interview items in the self-report screening questionnaire (e.g., thoughts about suicide, making plans or attempting suicide) [28]. Patients in both groups were allowed to use sleep medication and over-the-counter sleep aids without restrictions.

\section{General Practices}

At first, we recruited general practices through the Academic Network of General Practices $(n=8)$. This is a structural collaboration between a number of general practices in the Amsterdam area and the department of General Practice at Amsterdam University Medical Centre. Due to low inclusion rates, we sought additional practices outside Amsterdam during the trial $(n=7)$. There were around 50 GPs working in the 15 participating general practices.

The GP practices employed mental health nurses with either a university degree in psychology (62\%) or higher vocational training as a psychiatric nurse (38\%). Their average age was 51 (SD = 11, range 32-64 years) Their clinical experience ranged from 6 months to 15 years $($ mean $=6.9$ years, $S D=5)$. Most of them reported some experience with e-Health $(77 \%), 8 \%$ reported little experience and $15 \%$ no experience.

${ }^{1}$ Due to the low inclusion rates and difficulty reaching our envisioned sample size, we made several changes to the protocol along the course of the trial. First, we changed an exclusion criterion. Originally, the protocol was to exclude patients who had psychological treatment (for insomnia or other complaints) in the past 6 months. We switched to excluding only patients in current psychological treatment. Patients previously excluded for this reason $(n=3)$ were invited to participate again (of whom 2 did). Second, we initially recruited only patients who consulted their GP for insomnia prospectively. We switched to GPs actively screening electronic medical records and inviting all patients who had consulted their GP in the last year to our screening questionnaire. Third, we dropped actigraphy measures early on in the trial because these led to considerable logistic difficulties.

Internet-Delivered CBT-I in General

Practice: A Randomized Controlled Trial

\section{Intervention}

The i-Sleep intervention was developed by the first, second and last authors and is based on CBT-I [29-33]. i-Sleep consists of 5 lessons. Each lesson consists of information which is offered in text format as well as video. Furthermore, every lesson includes a fictitious and a real-life case example (also in text format and video). After every lesson the patient needs to fill out "homework" (sleep diary and e.g. setting bedtimes). Lesson 1 focused on psychoeducation about healthy and disordered sleep and sleep hygiene. In lesson 2, stimulus control is introduced (getting out of bed when awake for $>15 \mathrm{~min}$, using the bed for sleep and sexual activity only) and patients start sleep restriction therapy. Lesson 3 teaches relaxation and minimizing worrying. Lesson 4 is aimed at tackling dysfunctional cognitions about sleep. The fifth and final lesson invites the participant to make a summary of lessons learned and construct a relapse prevention plan.

Participants were advised to complete one lesson per week and received online coaching from the nurse in their own general practice. The aim of the feedback was to motivate patients to carry on, to offer explanations if needed and to comment on the completed exercises. Patients had the possibility to ask the nurses additional questions (online). Nurses received approximately $1.5 \mathrm{~h}$ of training from the first author (T.Z.) on how to guide i-Sleep and background knowledge about sleep and treatment. Every nurse first treated a mock patient under supervision (T.Z.), using the feedback manual created for this trial. Further supervision was offered on an as-needed basis, assessed by regular phone calls and e-mail contact (T.Z.). Nurses reported spending between 5 and 20 min per patient per session on providing online feedback. Feedback was programprompted: all participants received feedback on all completed sessions. The intervention did not include face-to-face contact and was provided at no cost to participants. Intervention group participants were free to receive other GP care as needed. The i-Sleep intervention is not primarily aimed at tapering or discontinuing medication but does discuss the (dis)advantages of medication. $\mathrm{Pa}$ tients are advised to contact their GP if they want to reduce or quit. A previous study has shown that the i-Sleep intervention is effective among insomnia patients recruited in the general population [21].

\section{CAU Control Condition}

GPs participating in this study received a printed version of the most recent Dutch insomnia guidelines for GPs [10] to promote a similar knowledge level on guidelines among participating practices. We did not otherwise interfere with CAU as offered by GPs. We reported to GPs which patients were randomized to CAU and invited these participants to use i-Sleep 6 months after randomization.

\section{Measurements}

Timing of the Assessments

Assessment took place at baseline (week 0), after treatment (week 8), at 26 weeks and 52 weeks. After the third assessment (26 weeks), the CAU patients were offered the i-Sleep treatment ( $n=$ 37 [57\%] accepted). The 52-week follow-up is uncontrolled. This follow-up aimed to determine whether effects in the intervention group are sustained over time.

\section{Primary Outcome Measures}

The primary outcome measure was the Insomnia Severity Index [34] (ISI; range 0-28). The ISI contains 7 items scored on a 
5 -point Likert scale ( $0=$ not at all, $4=$ extremely). The ISI is a reliable instrument possessing adequate internal consistency (Cronbach's $\alpha=0.90$ [35]) and was validated for online use [36]. Clinically significant improvement is defined as a change of $\geq 8$ points, a clinical cutoff of ISI $>10$ is used to define patients with clinical insomnia symptoms [35].

\section{Secondary Outcome Measures}

We used a pen-and-paper version of the Dutch translation of the Carney consensus diary [37] to estimate several sleep characteristics: sleep onset latency (SOL; number of minutes it takes to fall asleep), sleep efficiency (SE; 0-100\%; total sleep time divided by the total time in bed), number of awakenings (NWAK), wake after sleep onset (WASO; total minutes awake after sleep onset), terminal wakefulness (TWAK; time spent awake in bed before getting up), total sleep time (TST) and sleep quality (SQ; rated 1-5 each night). Participants completed 7-day diaries at each assessment, aggregated into week averages for analysis. Diaries with at least 5 days completed were included in the analysis.

We used the 14-item Hospital Anxiety and Depression Scale to assess anxiety and depression $[38,39]$. Each item is scored on a scale of $0-3$, and the total scores thus range from 0 to 21 for both the Anxiety and the Depression scales.

Daytime functioning (energy, relationships, mood, concentration, productivity and sleepiness) was measured using a 5-point scale (0-4; range 0-24) for each of the 6 items of the Daytime Consequences items [25]. We used the Work and Social Adjustment Scale to measure 4 domains of functioning (household tasks, work productivity, close relationships and social life) [40]. Each domain is measured on a scale of $0-8$. Fatigue was measured with the Fatigue Severity Scale [41] which consists of 7 items (scored 1-7). The total score ranges from 7 (no fatigue) to 49 (severely fatigued).

Finally, we assessed the use of sleep medication and antidepressant use. These data were collected both by direct reporting by participants and by examining the electronic medical records of the participants, separately. Due to privacy regulations we could not cross-check the medication use and therefore we report on both self-report (at the different assessments) and GP records (covering the complete inclusion period). From GP records we extracted whether patients had received at least one prescription during the assessment period (between baseline and follow-up at 26 weeks) of benzodiazepine derivatives (N05CD, N05BA), benzodiazepine-related drugs (N05CF) or antidepressants (N06A), using the Anatomical Therapeutic Chemical classification of the World Health Organization (www.whocc.no/atc). From these records we could not determine whether a patient's use had declined over the course of the trial, hence we additionally asked patients to report medication use at all assessments ("Have you used sleep medication in the past 4 weeks?" - yes/no).

Participants were asked to report (minor) falls, traffic accidents and other adverse events ("Did anything unpleasant happen to you that was related to your sleep or participation in the trial?") at each assessment. If yes, they were asked to describe what happened and whether any medical or psychological assistance was needed. ${ }^{2}$

\footnotetext{
${ }^{2}$ We did not report on data on alcohol use as per protocol due to a technical error in the data collection procedure that led to a lot of missing data. One of the originally 5 items of the Work and Social Adjustment Scale is missing for the same reason (we report on the other 4 and not on a total score).
}

\section{Statistical Analysis}

We performed intent-to-treat analyses (i.e., on all 134 included participants, irrespective of whether treatment was completed) [42]. We performed generalized mixed multilevel (patient and condition) regression analysis to evaluate the effects of time (within-group), condition (between-group) and the interaction between time $\times$ condition (between-group). For normally distributed outcomes regular linear mixed regressions using identity links were used, and mixed negative binomial regression analyses with a log link otherwise. To assess differences in change in outcomes between the two conditions, we performed a series of two-level (participants and time points) repeated-measures analyses in two separate models: one to assess change in outcome from pre- to posttest assessment and one assessing change in outcomes from pre- to 26 weeks follow-up. Assuming data were missing at random, we applied an unstructured covariance matrix as most fitting to the data [43]. Baseline characteristics significantly predicting dropout were added to the model as covariates (daytime consequences and sex). Baseline differences between the two conditions were not tested for statistical significance [44]. Between-group Cohen's $d$ effect sizes were calculated by calculating the pre-post change scores for both groups and calculating the difference. We then divided these by the pooled standard deviations observed at baseline [45].

We also compared the percentage of patients recovered (ISI below clinical cutoff of 11) or showing clinically significant improvement on the primary outcome (ISI; defined as a change of $\geq 8$ points) [35] between the intervention and control group at posttest examination and at 26 weeks follow-up. For the uncontrolled 52week follow-up, within-group effect sizes were calculated based on observed means. For all statistical analyses, the two-sided significance level was set at $p<0.05$. Data were analyzed using SPSS for Windows 25.

\section{Changes to Protocol}

We made two changes to our original protocol [22] both with the intention to enhance recruitment. In the original protocol the recruitment strategy was described as GPs inviting patients during a face-to-face consultation for sleep problems. We added a direct mailing of all patients who had consulted the GP for sleep problems in the last year. Furthermore, in the original protocol we described that we would exclude patients who received prior or current psychological treatment. We changed this to excluding patients only for current, not prior, psychological treatment.

\section{Results}

\section{Participants}

The final sample consisted of 134 adults, randomized to i-Sleep $(n=69)$ or the CAU control group $(n=65$; Fig. 1 for a flowchart). ${ }^{3}$ The mean age was $50.6(\mathrm{SD}=$

${ }^{3}$ Further details on the analysis are available from the first author (i.e., tables with regression coefficients, observed means and observed within-group effect sizes). 
Table 1. Characteristics of the sample at baseline

\begin{tabular}{lcc}
\hline Characteristic & $\begin{array}{l}\text { i-Sleep } \\
(n=69)\end{array}$ & $\begin{array}{l}\text { Control } \\
(n=65)\end{array}$ \\
\hline Age, years & $51.7(15.77)$ & $49.4(16.01)$ \\
Female sex & $43(62 \%)$ & $44(68 \%)$ \\
Higher education ${ }^{1}$ & $53(77 \%)$ & $39(60 \%)$ \\
Born in the Netherlands & $60(87 \%)$ & $56(86 \%)$ \\
Living situation & $22(32 \%)$ & $29(45 \%)$ \\
$\quad \begin{array}{l}\text { Alone } \\
\quad \text { Married/living together }\end{array}$ & $45(65 \%)$ & $31(48 \%)$ \\
$\quad$ Other & $2(3 \%)$ & $5(7 \%)$ \\
Living with children & $22(32 \%)$ & $15(23 \%)$ \\
Paid job or own company & $48(70 \%)$ & $45(69 \%)$ \\
Prescribed sleep medication use & $23(33 \%)$ & $25(39 \%)$ \\
Previous psychological treatment & & \\
$\quad$ (any) & $13(19 \%)$ & $16(25 \%)$ \\
\hline
\end{tabular}

Data expressed as means (standard deviation) or numbers (\%). ${ }^{1}$ Higher, higher vocational or university education.
15.9), $65 \%$ of the sample was female and $87 \%$ was born in the Netherlands (Table 1). In the i-Sleep condition, 47 participants $(68 \%)$ completed the intervention, and 22 (32\%) did not (8 did not start and 14 dropped out during treatment). The study response rate (providing data) was $83 \%(n=111)$ at the posttest assessment, $80 \%(n=107)$ at 26 weeks and $62 \%(n=43$, intervention group only) at 52 weeks. Contributions to data did not differ significantly between conditions ( 8 weeks: $\chi^{2}=0.28, p=0.60$; 26week follow-up: $\left.\chi^{2}=0.817, p=0.37\right)$. At baseline, the average insomnia score (ISI $=18.9$ ) refers to moderate clinical levels of insomnia. There were no patients without insomnia (as defined by ISI scores $<11$ ), 17 patients $(13 \%)$ reported subthreshold clinical insomnia (ISI $<14$ ), 80 reported moderate-severe (60\%; ISI 15-21) and 37 reported severe insomnia (28\%; ISI 22-28). At baseline, sleep medication was used by $33 \%$ (i-Sleep group) versus 39\% (control group) of the participants. The average score on anxiety was 8.16 which indicates subclinical levels of anxiety (>11 indicative of anxiety disorder) [38]. The average score on depression was 6.21 which indicates subclinical levels of depression ( $>11$ indicative of depressive disorder) [38]. Of all patients, 20 (15\%) scored above the anxiety cutoff, and 13 patients (10\%) scored above the depression cutoff [39].

\section{Primary Outcome: Effect on ISI}

Multilevel regression analyses showed significant time $\times$ condition interaction effects for the ISI, from pre-

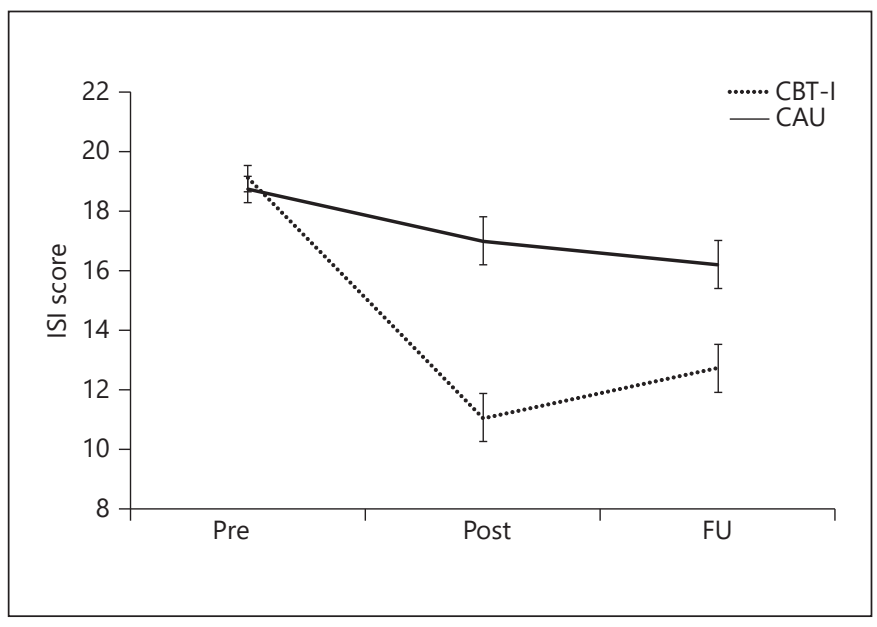

Fig. 2. Insomnia Severity Index (ISI) estimated means for intentto-treat analysis from baseline (pre) to posttest assessment and from baseline to 26-week and 52-week follow-up (FU). to posttest assessment, $F_{1,88}=29.62, p<0.001, d_{\text {between }}=$ 1.66 and from pre-examination to follow-up at 26 weeks, $F_{1,74}=10.65, p=0.002, d_{\text {between }}=1.02$ (Fig. 2). This indicates that I-CBT-I resulted in participants experiencing a greater improvement in insomnia severity than CAU patients from pre- to postassessment and from preassessment to 26-week follow-up. See Table 2 for confidence intervals. The intervention group more often reported a clinically significant improvement (improvement on ISI score $\geq 8$ points) than patients in the control group at the posttest assessment (56 vs. 12\%) and at 26 weeks (46 vs. $11 \%$; Table 3).

\section{Secondary Outcomes: Effect on Sleep Diary Indices}

Multilevel regression analyses showed significant time $\times$ condition interaction effects at the posttest assessment for the sleep diary variables: WASO, $F_{1,94}=14.09$, $p<0.001, d_{\text {between }}=0.54$; NWAK, $F_{1,102}=11.32, p \leq$ $0.001, d_{\text {between }}=0.43$; TWAK, $F_{1,96}=5.84, p=0.018$, $d_{\text {between }}=0.31$; SQ, $F_{1,90}=5.23, p=0.025, d_{\text {between }}=0.52$ and SE, $F_{1,112}=17.23, p<0.001, d_{\text {between }}=0.61$. This indicates that participants in the i-Sleep condition showed more improvement on time spent awake in bed and sleep efficiency than patients receiving CAU at the posttest assessment. No significant differences were observed for SOL, TST or sleep quality. At 26 weeks of follow-up, significant interaction effects of time $\times$ condition were observed for SE, $F_{1,103}=5.55, p=0.02, d_{\text {between }}=0.41$, and TST, $F_{1,99}=5.89, p=0.017, d_{\text {between }}=0.41$ (Table 2). 
Table 2. Multilevel intention-to-treat regression based pre-, post- and follow-up (FU) estimates and Cohen's $d$ effect sizes

\begin{tabular}{|c|c|c|c|c|c|c|c|c|c|}
\hline \multirow[t]{2}{*}{ Measure } & \multirow[t]{2}{*}{ Group } & \multicolumn{2}{|c|}{$\begin{array}{l}\text { Pre-post model-estimated mean score } \\
\text { (StE) }\end{array}$} & \multicolumn{2}{|c|}{$\begin{array}{l}\text { Pre-FU model-estimated mean score } \\
\text { (StE) }\end{array}$} & \multirow{2}{*}{$\begin{array}{l}\text { Between } \\
\text { Cohen's } d^{1} \\
\text { effect size: } \\
\text { pre to post }\end{array}$} & \multirow[t]{2}{*}{ Significance $^{2}$} & \multirow{2}{*}{$\begin{array}{l}\text { Between } \\
\text { Cohen's } d \\
\text { effect size: } \\
\text { pre to FU }\end{array}$} & \multirow[t]{2}{*}{ Significance } \\
\hline & & Pre & Post & Pre & Post & & & & \\
\hline \multirow[t]{2}{*}{ ISI } & i-Sleep & $19.12(0.42)$ & $11.04(0.81)$ & $19.26(0.44)$ & $12.73(0.88)$ & 1.66 & $p<0.001$ & 1.02 & $p=0.002$ \\
\hline & CAU & $18.74(0.47)$ & $16.99(0.74)$ & $18.85(0.46)$ & $16.20(0.81)$ & & & & \\
\hline \multirow[t]{2}{*}{ SOL } & i-Sleep & $46.99(4.15)$ & $31.41(3.10)$ & $47.37(4.01)$ & $36.17(3.56)$ & 0.22 & $p=0.099$ & 0.21 & $p=0.190$ \\
\hline & CAU & $45.89(5.60)$ & $39.67(4.10)$ & $44.71(5.58)$ & $42.51(5.31)$ & & & & \\
\hline \multirow[t]{2}{*}{ WASO } & i-Sleep & $54.98(4.86)$ & $30.25(4.22)$ & $55.61(5.08)$ & $33.90(4.76)$ & 0.54 & $p<0.001$ & 0.10 & $p=0.435$ \\
\hline & $\mathrm{CAU}$ & $57.39(6.13)$ & $56.92(6.84)$ & $57.01(5.87)$ & $39.85(5.30)$ & & & & \\
\hline \multirow[t]{2}{*}{ NWAK } & i-Sleep & $1.27(0.15)$ & $0.85(0.13)$ & $1.28(0.15)$ & $0.98(0.16)$ & 0.43 & $p=0.001$ & 0.10 & $p=0.575$ \\
\hline & $\mathrm{CAU}^{\circ}$ & $1.15(0.15)$ & $1.23(0.16)$ & $1.15(0.15)$ & $0.97(0.15)$ & & & & \\
\hline \multirow[t]{2}{*}{ TWAK } & i-Sleep & $45.67(3.23)$ & $25.59(3.05)$ & $45.78(3.15)$ & $30.32(4.45)$ & 0.31 & $p=0.018$ & 0.28 & $p=0.121$ \\
\hline & CAU & $42.99(4.27)$ & $33.92(3.76)$ & $43.93(4.44)$ & $38.55(5.20)$ & & & & \\
\hline \multirow[t]{2}{*}{ TST } & i-Sleep & $341.41(6.63)$ & $372.49(7.61)$ & $339.60(6.74)$ & $382.02(7.11)$ & 0.23 & $p=0.122$ & 0.41 & $p=0.017$ \\
\hline & CAU & $357.04(8.17)$ & $372.66(9.35)$ & $354.87(8.06)$ & $369.97(9.94)$ & & & & \\
\hline \multirow[t]{2}{*}{ SE } & i-Sleep & $66.84(1.40)$ & $77.64(1.33)$ & $66.37(1.42)$ & $76.26(1.56)$ & 0.61 & $p<0.001$ & 0.41 & $p=0.020$ \\
\hline & $\mathrm{CAU}^{\circ}$ & $68.86(1.68)$ & $71.59(1.83)$ & $68.59(1.72)$ & $73.08(2.05)$ & & & & \\
\hline \multirow[t]{2}{*}{ Sleep quality } & i-Sleep & $2.50(0.07)$ & $2.87(0.07)$ & $2.53(0.08)$ & $3.11(0.08)$ & 0.52 & $p=0.025$ & 0.40 & $p=0.103$ \\
\hline & CAU & $2.65(0.07)$ & $2.71(0.10)$ & $2.67(0.07)$ & $3.01(0.11)$ & & & & \\
\hline \multirow[t]{2}{*}{ Daytime cons. } & i-Sleep & $18.62(0.42)$ & $16.04(0.59)$ & $18.66(0.43)$ & $16.18(0.61)$ & 0.39 & $p=0.123$ & 0.26 & $p=0.248$ \\
\hline & CAU & $19.81(0.41)$ & $18.55(0.53)$ & $19.87(0.41)$ & $18.26(0.50)$ & & & & \\
\hline \multirow[t]{2}{*}{ HADS-A } & i-Sleep & $8.47(0.37)$ & $7.55(0.44)$ & $8.41(0.36)$ & $7.54(0.43)$ & 0.19 & $p=0.275$ & 0.15 & $p=0.309$ \\
\hline & CAU & $8.07(0.36)$ & $7.70(0.43)$ & $7.92(0.35)$ & $7.49(0.37)$ & & & & \\
\hline \multirow[t]{2}{*}{ HADS-D } & i-Sleep & $6.13(0.35)$ & $4.59(0.50)$ & $6.10(0.38)$ & $4.50(0.50)$ & 0.37 & $p=0.024$ & 0.21 & $p=0.154$ \\
\hline & CAU & $5.85(0.48)$ & $5.77(0.46)$ & $5.78(0.48)$ & $5.00(0.45)$ & & & & \\
\hline \multirow[t]{2}{*}{ Fatigue (FSS) } & i-Sleep & $39.51(0.83)$ & $33.76(1.27)$ & $39.54(0.83)$ & $33.60(1.46)$ & 0.08 & $p=0.641$ & 0.10 & $p=0.567$ \\
\hline & $\mathrm{CAU}^{\circ}$ & $40.39(1.14)$ & $35.45(1.49)$ & $40.48(1.13)$ & $33.53(1.49)$ & & & & \\
\hline \multirow[t]{2}{*}{ WSAS: household } & i-Sleep & $3.66(0.23)$ & $2.72(0.29)$ & $3.53(0.22)$ & $3.22(0.26)$ & 0.32 & $p=0.108$ & 0.03 & $p=0.879$ \\
\hline & CAU & $4.20(0.25)$ & $3.92(0.27)$ & $4.07(0.25)$ & $3.70(0.33)$ & & & & \\
\hline \multirow[t]{2}{*}{ WSAS: work productivity } & i-Sleep & $4.51(0.18)$ & $2.84(0.28)$ & $4.46(0.18)$ & $3.63(0.32)$ & 0.38 & $p=0.097$ & 0.09 & $p=0.714$ \\
\hline & $\mathrm{CAU}^{1}$ & $4.54(0.22)$ & $3.58(0.25)$ & $4.50(0.23)$ & $3.50(0.30)$ & & & & \\
\hline \multirow[t]{2}{*}{ WSAS: close relationships } & i-Sleep & $3.86(0.21)$ & $2.90(0.29)$ & $3.76(0.21)$ & $3.42(0.33)$ & 0.21 & $p=0.247$ & 0.11 & $p=0.609$ \\
\hline & $\mathrm{CAU}$ & $3.99(0.24)$ & $3.46(0.24)$ & $3.91(0.23)$ & $3.35(0.26)$ & & & & \\
\hline \multirow[t]{2}{*}{ WSAS: social life } & i-Sleep & $4.26(0.22)$ & $3.08(0.28)$ & $4.14(0.23)$ & $3.51(0.32)$ & 0.36 & $p=0.052$ & 0.02 & $p=0.939$ \\
\hline & $\mathrm{CAU}$ & $4.11(0.23)$ & $3.69(0.24)$ & $4.02(0.23)$ & $3.43(0.26)$ & & & & \\
\hline
\end{tabular}

DC, daytime consequences; FU, follow-up; FSS, Fatigue Severity Scale; HADS-A, Hospital Anxiety and Depression Scale - anxiety subscale; i-Sleep, i-Sleep treatment condition; ISI, Insomnia Severity Index; NWAK, number of awakenings; SE, sleep efficiency; SOL, sleep onset latency; StE, standard error; TST, total sleep time; TWAK, terminal wakefulness; WASO, wake after sleep onset; WSAS, work and social adjustment scale. ${ }^{1}$ Computed by dividing the difference in posttest scores by the pooled observed standard deviations at baseline; a Cohen's $d$ is commonly considered to be either small (i.e. $<0.20$ ), moderate (around 0.50 ) or large $\left(0.80\right.$ and over; Cohen [23]). Negative effect sizes indicate negative changes (i.e., higher severity of complaints, or CAU outperforming i-Sleep condition for between-groups effect size). ${ }^{2}$ Significance levels arge (0.80 and over, Cohen [23]). Negative eft

Table 3. $\chi^{2}$ tests of differences in clinical improvement on observed insomnia severity at posttest (8 weeks), 26-week and 52-week followup assessments

\begin{tabular}{|c|c|c|c|c|c|c|}
\hline & \multicolumn{2}{|l|}{ Post } & \multicolumn{2}{|c|}{ FU 26 weeks } & \multicolumn{2}{|c|}{ FU 52 weeks } \\
\hline & $\begin{array}{l}\text { recovered } \\
(\text { ISI < 11) }\end{array}$ & $\begin{array}{l}\text { clin. significantly } \\
\text { improved } \\
(\Delta \mathrm{ISI} \geq 8)\end{array}$ & $\begin{array}{l}\text { recovered } \\
(\text { ISI < 11) }\end{array}$ & $\begin{array}{l}\text { clin. significantly } \\
\text { improved } \\
(\Delta \mathrm{ISI} \geq 8)\end{array}$ & $\begin{array}{l}\text { recovered } \\
(\mathrm{ISI}<11)\end{array}$ & $\begin{array}{l}\text { clin. significantly } \\
\text { improved } \\
(\Delta \mathrm{ISI} \geq 8)\end{array}$ \\
\hline i-Sleep & $28(65 \%)$ & $24(56 \%)$ & $15(43 \%)$ & $16(46 \%)$ & $18(45 \%)$ & $16(40 \%)$ \\
\hline CAU & $2(5 \%)$ & $5(12 \%)$ & $3(8 \%)$ & $4(11 \%)$ & n.a. & n.a. \\
\hline$\chi^{2}$ & $33.2^{* * *}$ & $17.7^{* * *}$ & $11.6^{* * *}$ & $10.9^{* * *}$ & n.a. & n.a. \\
\hline
\end{tabular}

*** $p<0.001$. FU, follow-up; CAU, care as usual; ISI, Insomnia Severity Index; n.a., not available.

Secondary Outcomes: Effect on Mood, Anxiety,

Fatigue and Daily Functioning

Multilevel regression analyses showed a significant time $\times$ condition interaction effect at the posttest assess- ment for depression, $F_{1,103}=5.28, p=0.024, d_{\text {between }}=0.37$, but no statistically significant time $\times$ condition effects on anxiety, fatigue, daytime consequences or daily functioning (Work and Social Adjustment Scale items: household 
tasks, work productivity, close relationships and social life). At 26 weeks of follow-up, no significant interaction effects were observed for any of the secondary outcomes. Both conditions improved significantly over time on these measures of mental health and daytime functioning.

\section{Secondary Outcomes: Medication}

There were no significant differences in sleep medication use, as reported by patients themselves, between the intervention and CAU group at baseline (33 vs. $39 \%, p=$ 0.54 ) or at the posttest assessment ( 20 vs. $23 \% p=0.96$ ). At the 26-week follow-up, twice as many patients in the control group used medication (29\%) than in the intervention group (13\%). However, this difference is not statistically significant $(p=0.75)$. At the 52 -week follow-up, 10 i-Sleep participants (23\%) used sleep medication. GP records showed that over the complete trial period $30 \%$ used sleep medication ( $25 \%$ benzodiazepine derivatives, $5 \% \mathrm{Z}$ drugs) in the i-Sleep condition versus $47 \%$ of the control group (40\% benzodiazepine derivatives, $7 \% \mathrm{Z}$ drugs). Antidepressants were used by $12 \%$ of i-Sleep participants over this period, versus $25 \%$ in the control group.

\section{One-Year Uncontrolled Follow-Up}

Within-group effect sizes in the i-Sleep condition indicate that the effects were sustained at 52 weeks of follow-up ( $d_{\text {within }}$ ranging from 1.69 [ISI] to 0.23 [SOL]); however, the response rate at 52 weeks of follow-up was quite low (62\%).

\section{Adverse Events}

No serious adverse events (warranting psychological or medical care) were reported. At the posttest assessment, one CAU participant mentioned a surgery for unrelated medical reasons.

\section{Discussion}

This pragmatic randomized controlled trial showed that nurse-guided I-CBT-I is effective in general practice in reducing insomnia symptoms. The study showed that I-CBT-I is feasible and can be used in general practice with support from nurses. The intervention yielded large effect sizes compared to CAU on insomnia severity ( $d=$ 1.66 after treatment, and $d=1.02$ after 26 weeks). These effects remained at the 52-week follow-up. We also demonstrated significant secondary effects at the posttest assessment (sleep diary WASO, NWAK, TWAK, SQ, and depressive symptoms) and 26-week follow-up (TST and

Internet-Delivered CBT-I in General

Practice: A Randomized Controlled Trial
SE). No significant effects were observed on the other secondary outcomes, anxiety, fatigue, daytime consequences or sleep medication.

Our aim was to test whether I-CBT-I's effects would stand the test in a general practice population. One of our hypotheses was that patients actively seeking help from their GP would be different than those who respond to online advertisement for insomnia treatment. However, when comparing the baseline scores of our population to those of other studies among the general population we do not see many differences. The participants in our trial were suffering from relatively severe insomnia (mean baseline ISI score 18.9 in our sample) but the same is the case in, for example, the online study of Kaldo et al. [46] (baseline ISI score 16.8) or Lancee et al. [47] (baseline ISI score 18.2 ). Baseline sleep efficiency (66\% in our trial) was also very similar to the ones reported by Kaldo et al. [46] (71\%) and Lancee et al. [47] (64\%). Furthermore, 15\% of our population scored above the cutoff on our anxiety questionnaire and $10 \%$ did so for depression. This also seems to indicate that our population did not suffer substantially more often from comorbid mental disorders than those who are recruited in the general population. All this does not necessarily mean that people who actively seek help are similar to those who do not. They might be different on aspects we did not measure, e.g. the extent of social support, or their personality traits. It is important that we showed that I-CBT-I can be effectively delivered in general practice because in many countries the GP functions as a gatekeeper and is the first person to go to when looking for help for insomnia. This online intervention enables GPs to offer insomnia care according to the current clinical guidelines. Support costs relatively little time (on average $1.5 \mathrm{~h}$ per patient) and does not have to be delivered by highly trained (psychological) professionals but can be offered by people with a higher vocational background after a short training: in this case support was effectively executed by the nurses employed in the general practice.

We included not only sleep outcomes, but also other relevant secondary outcomes. Surprisingly, on many of these indices no effect of I-CBT-I was observed, a finding only partly in line with other trials. For instance, CBT-I often also has beneficial effects for depression and anxiety complaints [48-50]. We were able to replicate the effect at the posttest assessment for depressive complaints but this was no longer detected at the 26-week follow-up. The relatively low level of severity of depression in our sample might explain the lack of mental health effects. Our sample may have consisted of people with an isolated insomnia problem not

Psychother Psychosom 2020;89:174-184 181 
(yet) generalized to other areas of mental health (i.e., only $10 \%$ suffered from depression). Our participants did show considerable baseline impairments in daytime functioning. Most studies observe improvements in daytime functioning after insomnia treatment $[20,25]$. We did not find a significant effect but the effect sizes for the various domains of functioning are between $d=0.21$ and $d=0.39$. A possible explanation for the lack of significance is that the study was underpowered to detect these clinically relevant effects, although by adding baseline measurements to the analysis we have likely increased power [51].

It remains difficult to understand that the intervention showed large effects on insomnia but not on fatigue. Fatigue is one of the most frequently reported symptoms of patients seeking help from insomnia. However, a recent meta-analysis into the effect of insomnia treatments on fatigue also concludes that there is no effect [50]. Importantly, this meta-analysis indicates that there is a high heterogeneity between studies indicating that some are more successful than others. At the moment, the association between sleep and fatigue is still poorly understood. We definitely need more research to uncover the (un)shared mechanisms. Network intervention analysis could be used in order to shed light on the treatment effects on individual symptoms, since insomnia, fatigue and daytime symptom questionnaires are prone to overlap somewhat [52].

The assessments of sleep medication and antidepressant use were complicated, due to the combination of selfreport and GP data which we could not link. Moreover, a prescription is not necessarily the same as usage. Using a more reliable measure (such as sophisticated bottle cap chips counting numbers of pills taken) was not feasible in this trial. Even though there are no significant differences, both the GP data and the self-report data indicate less use of sleep medication (and antidepressants) in the intervention group than in the control group. At 26 weeks the control group reports more than twice $(29 \%)$ as much sleep medication use than the intervention group (13\%), which is a promising result which needs to be replicated in larger trials. Previous research has indeed indicated that CBT-I can have favorable effects on sleep medication use and can facilitate the discontinuation process [53].

Our trial has several strong features. First of all, that it was a pragmatic trial. We showed that it is feasible to implement an I-CBT-I treatment in routine GP care. Furthermore, we showed that the treatment is acceptable for patients. A large majority (68\%) completed the treatment fully, which is higher than often observed in online treatment for insomnia [24]. Adherence might even be raised by adding a face-to-face session before the start of the treatment or by offering it in a blended way (online and face-to-face sessions). This might depend on the individual patients' motivation and other characteristics. Another strong feature is our high assessment response rate (Fig. 1). About $80 \%$ completed the posttest measurement.

A limitation of our study is the slow inclusion rate and the early termination of inclusion before we reached our desired sample size which has consequences for the power of the secondary analyses in particular. The low inclusion rate was partly due to time and resource constraints in general practice. Although the participating GPs and nurses were highly motivated and enthusiastic about having an alternative treatment to offer insomnia patients other than or in addition to medication, the trial was not always mentioned during (insomnia-related) GP consultations which may have lowered inclusion rates. GP awareness and involvement should be a strong focus point in future research. An additional limitation is that polysomnographic measurements were not feasible in this research setting. Although we deem subjective measures of this psychological problem to be most important, as is reflected by the DSM-5 nosology [26], it would have been valuable to have polysomnographic data in addition. We aimed to include actigraphy data, but unfortunately this also turned out not to be feasible. The research context likely influenced uptake as well. Patients may have been hesitant to take part in a trial and could be more willing to undergo I-CBT-I through their GP outside of the research setting. A related limitation is the use of a CAU control condition [54]. We had expected that GPs would have provided more care (e.g., medication, sleep hygiene advice, referral to a psychologist or physiotherapist) since we explicitly handed over the most recent insomnia guidelines and notified the GPs of the patients who were randomized to the control group. However, it turned out that most patients in the control group did not receive much care and started i-Sleep after the waiting period. The control group thus resembled a waitlist condition which may have induced a nocebo effect [55].

In summary, we reached our goal of offering GPs a time-efficient treatment that is highly effective in reducing insomnia complaints. A cost-effectiveness analysis of the intervention will be a valuable next step, as would comparing the intervention to an unguided version offered in general practice. This might increase uptake since it requires even less work by GPs and nurses, but it may decrease adherence and have a lower effect [17]. We demonstrated the potential of I-CBT-I in routine general practice: i-Sleep clearly outperformed CAU on insomnia severity and several sleep diary indices in this trial. The 
large effects on insomnia severity are important towards prevention of mental health problems for which insomnia is a risk factor as well [4]. Although further research is needed to illuminate potential effects on other mental health complaints and daily functioning and the longerterm effects of online interventions [56], we recommend GPs to implement nurse-guided I-CBT-I as a way of alleviating insomnia symptoms through feasible, effective, guideline adherent treatment.

\section{Acknowledgments}

We thank all participating patients, GPs and nurses for their time and effort.

\section{Statement of Ethics}

The authors assert that all procedures contributing to this work comply with the ethical standards of the relevant national and institutional committees on human experimentation and with the Helsinki Declaration of 1975, as revised in 2008.

\section{Disclosure Statement}

J.E.B., E.J.W.S. and P.S. have no conflicts of interest to declare. A.S., J.L. and T.Z. have developed the online treatment and made the book version available online (PDF) and in print, but have no commercial interests.

\section{Funding Sources}

The trial was funded by ZonMW (project No.: 837002504).

\section{Author Contributions}

T.Z., A.S., J.L., E.J.W.S. and P.S. contributed to the design of the study. A.S. and J.L. wrote the i-Sleep treatment manual. T.Z. developed the Internet-delivered i-Sleep intervention. T.Z. carried out recruitment and data collection, supervised the treatment delivery and provided the guidance for control participants after the controlled phase of the trial. T.Z. performed the statistical analysis and drafted the manuscript. All authors contributed to and approved the paper.

\section{References}

1 Ohayon MM. Epidemiology of insomnia: what we know and what we still need to learn. Sleep Med Rev. 2002 Apr;6(2):97-111.

2 Wittchen HU, Jacobi F, Rehm J, Gustavsson A, Svensson M, Jönsson B, et al. The size and burden of mental disorders and other disorders of the brain in Europe 2010. Eur Neuropsychopharmacol. 2011 Sep;21(9):655-79.

3 Van der Linden MW, Westert GP, de Bakker DH, Schellevis FG. Tweede Nationale Studie naar ziekten en verrichtingen in de huisartspraktijk. Klachten en aandoeningen in de bevolking en in de huisartspraktijk. Utrecht, Bilthoven: NIVEL/RIVM; 2004.

4 Li MJ, Kechter A, Olmstead RE, Irwin MR, Black DS. Sleep and mood in older adults: coinciding changes in insomnia and depression symptoms. Int Psychogeriatr. 2018 Mar; 30(3):431-5.

5 Olfson M, Wall M, Liu SM, Morin CM, Blanco C. Insomnia and Impaired Quality of Life in the United States. J Clin Psychiatry. 2018 Sep;79(5):17m12020.

6 Sofi F, Cesari F, Casini A, Macchi C, Abbate $\mathrm{R}$, Gensini GF. Insomnia and risk of cardiovascular disease: a meta-analysis. Eur J Prev Cardiol. 2014 Jan;21(1):57-64

7 Daley M, Morin CM, LeBlanc M, Grégoire JP, Savard J. The economic burden of insomnia: direct and indirect costs for individuals with insomnia syndrome, insomnia symptoms, and good sleepers. Sleep. 2009a Jan;32(1):55-64.
8 Daley M, Morin CM, LeBlanc M, Grégoire JP, Savard J, Baillargeon L. Insomnia and its relationship to health-care utilization, work absenteeism, productivity and accidents. Sleep Med. 2009b Apr;10(4):427-38.

9 Van Straten A, van der Zweerde T, Kleiboer A, Cuijpers P, Morin CM, Lancee J. Cognitive and behavioral therapies in the treatment of insomnia: a meta-analysis. Sleep Med Rev. 2018 Apr;38:3-16.

10 NHG-werkgroep Slaapproblemen en slaapmiddelen. NHG-Standaard Slaapproblemen en slaapmiddelen (tweede herziening). Huisarts Wet. 2014;57:352-61.

11 National Institute for Clinical Excellence. Guidance on the use of zaleplon, zolpidem and zopiclone for the short-term management of insomnia. Technology Appraisal Guidance [TA77]. London: NICE; 2004.

12 Qaseem A, Kansagara D, Forciea MA, Cooke M, Denberg TD; Clinical Guidelines Committee of the American College of Physicians. Management of chronic insomnia disorder in adults: a clinical practice guideline from the American College of Physicians. Ann Intern Med. 2016 Jul;165(2):125-33.

13 Riemann D, Baglioni C, Bassetti C, Bjorvatn B, Dolenc Groselj L, Ellis JG, et al. European guideline for the diagnosis and treatment of insomnia. J Sleep Res. 2017 Dec;26(6):675700 .
14 Cheung JM, Jarrin DC, Ballot O, Bharwani AA, Morin CM. A systematic review of cognitive behavioral therapy for insomnia implemented in primary care and community settings. Sleep Med Rev. 2019 Apr;44:23-36.

15 Hoebert J, Souverein P, Mantel-Teeuwisse AK, Leufkens HG, van Dijk L. Benzodiazepinegebruik daalt als de gebruiker zelf betaalt. Huisarts Wet. 2012 Jul;55(7):286-8.

16 Everitt H, McDermott L, Leydon G, Yules H, Baldwin D, Little P. GPs' management strategies for patients with insomnia: a survey and qualitative interview study. Br J Gen Pract. 2014 Feb;64(619):e112-9.

17 Lancee J, van den Bout J, Sorbi MJ, van Straten A. Motivational support provided via email improves the effectiveness of internet-delivered self-help treatment for insomnia: a randomized trial. Behav Res Ther. 2013 Dec; 51(12):797-805.

18 Andersson G, Cuijpers P, Carlbring P, Riper $\mathrm{H}$, Hedman E. Guided Internet-based vs. face-to-face cognitive behavior therapy for psychiatric and somatic disorders: a systematic review and meta-analysis. World Psychiatry. 2014 Oct;13(3):288-95.

19 Zachariae R, Lyby MS, Ritterband LM, O’Toole MS. Efficacy of internet-delivered cognitive-behavioral therapy for insomnia: a systematic review and meta-analysis of randomized controlled trials. Sleep Med Rev. 2016 Dec;30:1-10.
Internet-Delivered CBT-I in General

Practice: A Randomized Controlled Trial
Psychother Psychosom 2020;89:174-184 DOI: $10.1159 / 000505600$ 
20 Espie CA, Emsley R, Kyle SD, Gordon C, Drake CL, Siriwardena AN, et al. Effect of digital cognitive behavioral therapy for insomnia on health, psychological well-being, and sleep-related quality of life: a randomized clinical trial. JAMA Psychiat. 2019 Jan;76(1): 21-30.

21 van Straten A, Emmelkamp J, de Wit J, Lancee J, Andersson G, van Someren EJ, et al. Guided Internet-delivered cognitive behavioural treatment for insomnia: a randomized trial. Psychol Med. 2014 May;44(7):1521-32.

22 Van der Zweerde T, Lancee J, Slottje P, Bosmans J, Van Someren E, Reynolds C 3rd, et al. Cost-effectiveness of i-Sleep, a guided online CBT intervention, for patients with insomnia in general practice: protocol of a pragmatic randomized controlled trial. BMC Psychiatry. 2016 Apr;16(Dec):85.

23 Cohen J. Statistical Power Analysis for the Behavioral Sciences. 2nd ed. New York: Lawrence Erlbaum Associates; 1988.

24 Horsch C, Lancee J, Beun RJ, Neerincx MA, Brinkman WP. Adherence to technology-mediated insomnia treatment: a meta-analysis, interviews, and focus groups. J Med Internet Res. 2015 Sep;17(9):e214.

25 Espie CA, Kyle SD, Williams C, Ong JC, Douglas NJ, Hames P, et al. A randomized, placebo-controlled trial of online cognitive behavioral therapy for chronic insomnia disorder delivered via an automated media-rich web application. Sleep (Basel). 2012 Jun; 35(6):769-81.

26 American Psychiatric Association. Diagnostic and statistical manual of mental disorders. 5th ed. Washington: American Psychiatric Association; 2013.

27 Wilson SJ, Nutt DJ, Alford C, Argyropoulos SV, Baldwin DS, Bateson AN, et al. British Association for Psychopharmacology consensus statement on evidence-based treatment of insomnia, parasomnias and circadian rhythm disorders. J Psychopharmacol. 2010 Nov; 24(11):1577-601.

28 Sheehan DV, Lecrubier Y, Sheehan KH, Amorim P, Janavs J, Weiller E, et al. The Mini-International Neuropsychiatric Interview (M.I.N.I.): the development and validation of a structured diagnostic psychiatric interview for DSM-IV and ICD-10. J Clin Psychiatry. 1998;59 Suppl 20:22-33.

29 Edinger JD, Wohlgemuth WK. The significance and management of persistent primary insomnia: the past, present and future of behavioral insomnia therapies. Sleep Med Rev. 1999 Jun;3(2):101-18.

30 Morin CM, Espie CA. Insomnia: A Clinical Guide to Assessment and Treatment. New York: Kluwer Academic/Plenum Publishers; 2003.
31 Edinger JD, Means MK. Cognitive-behavioral therapy for primary insomnia. Clin Psychol Rev. 2005 Jul;25(5):539-58.

32 Verbeek I, Klip E. Insomnia. Amsterdam: Boom Uitgevers; 2005.

33 Espie C. Overcoming Insomnia and Sleep Problems: A Self-help Guide using Cognitive Behavioral Techniques. London: Robinson; 2006.

34 Bastien CH, Vallières A, Morin CM. Validation of the Insomnia Severity Index as an outcome measure for insomnia research. Sleep Med. 2001 Jul;2(4):297-307.

35 Morin CM, Belleville G, Bélanger L, Ivers $\mathrm{H}$. The Insomnia Severity Index: psychometric indicators to detect insomnia cases and evaluate treatment response. Sleep (Basel). 2011 May;34(5):601-8.

36 Thorndike FP, Ritterband LM, Saylor DK, Magee JC, Gonder-Frederick LA, Morin CM. Validation of the insomnia severity index as a web-based measure. Behav Sleep Med. 2011; 9(4):216-23.

37 Carney CE, Buysse DJ, Ancoli-Israel S, Edinger JD, Krystal AD, Lichstein KL, et al. The consensus sleep diary: standardizing prospective sleep self-monitoring. Sleep (Basel). 2012 Feb;35(2):287-302.

38 Olssøn I, Mykletun A, Dahl AA. The Hospital Anxiety and Depression Rating Scale: a crosssectional study of psychometrics and case finding abilities in general practice. BMC Psychiatry. 2005 Dec;5(1):46.

39 Spinhoven P, Ormel J, Sloekers PP, Kempen GI, Speckens AE, Van Hemert AM. A validation study of the Hospital Anxiety and Depression Scale (HADS) in different groups of Dutch subjects. Psychol Med. 1997 Mar; 27(2):363-70

40 Mundt JC, Marks IM, Shear MK, Greist JH. The Work and Social Adjustment Scale: a simple measure of impairment in functioning. Br J Psychiatry. 2002 May; 180(5):461-4.

41 Krupp LB, LaRocca NG, Muir-Nash J, Steinberg AD. The fatigue severity scale. Application to patients with multiple sclerosis and systemic lupus erythematosus. Arch Neurol. 1989 Oct;46(10):1121-3.

42 Newell DJ. Intention-to-treat analysis: implications for quantitative and qualitative research. Int J Epidemiol. 1992 Oct;21(5):83741.

43 Heck RH, Tabata L, Thomas SL. Multilevel and longitudinal modeling with IBM SPSS. Abingdon: Routledge; 2013. https://doi. org/10.4324/9780203701249.

44 Twisk JWR, Bosman L, Hoekstra T, Rijnhart JJM, Welten M, Heijmans M. Different ways to estimate treatment effects in randomised controlled trials. Contemp Clin Trials Commun. 2018 Mar; 10:80-5.

45 Morris SB. Estimating effect sizes from pretest-posttest-control group designs. Organ Res Methods. 2008 Apr;11(2):364-86.
46 Kaldo V, Jernelöv S, Blom K, Ljótsson B, Bro$\operatorname{din} M$, Jörgensen $M$, et al. Guided internet cognitive behavioral therapy for insomnia compared to a control treatment: a randomized trial. Behav Res Ther. 2015 Aug;71:90100 .

47 Lancee J, van Straten A, Morina N, Kaldo V, Kamphuis JH. Guided online or face-to-face cognitive behavioral treatment for insomnia: a randomized wait-list controlled trial. Sleep (Basel). 2016 Jan;39(1):183-91.

48 van der Zweerde T, van Straten A, Effting M, Kyle SD, Lancee J. Does online insomnia treatment reduce depressive symptoms? A randomized controlled trial in individuals with both insomnia and depressive symptoms. Psychol Med. 2019 Feb;49(3):501-9.

49 Ye YY, Zhang YF, Chen J, Liu J, Li XJ, Liu YZ, et al. Internet-based cognitive behavioral therapy for insomnia (ICBT-i) improves comorbid anxiety and depression-a metaanalysis of randomized controlled trials. PLoS One. 2015 Nov; 10(11):e0142258.

50 Ballesio A, Aquino MR, Feige B, Johann AF, Kyle SD, Spiegelhalder K, et al. The effectiveness of behavioural and cognitive behavioural therapies for insomnia on depressive and fatigue symptoms: a systematic review and network meta-analysis. Sleep Med Rev. 2018 Feb; 37:114-29.

51 Mazumdar S, Tang G, Houck PR, Dew MA, Begley AE, Scott J, et al. Statistical analysis of longitudinal psychiatric data with dropouts. J Psychiatr Res. 2007 Dec;41(12):1032-41.

52 Blanken TF, Van Der Zweerde T, Van Straten A, Van Someren EJ, Borsboom D, Lancee J. Introducing Network Intervention Analysis to investigate sequential, symptom-specific treatment effects: A demonstration in co-occurring insomnia and depression. Psychother Psychosom. 2019;88(1):52-4.

53 Ong J, Suh S. Utilizing cognitive-behavioral therapy for insomnia to facilitate discontinuation of sleep medication in chronic insomnia patients. Sleep Med Res. 2012 Aug;3(1):1-6.

54 Guidi J, Brakemeier EL, Bockting CL, Cosci F, Cuijpers P, Jarrett RB, et al. Methodological recommendations for trials of psychological interventions. Psychother Psychosom. 2018; 87(5):276-84.

55 Furukawa TA, Noma H, Caldwell DM, Honyashiki M, Shinohara K, Imai $\mathrm{H}$, et al. Waiting list may be a nocebo condition in psychotherapy trials: a contribution from network metaanalysis. Acta Psychiatr Scand. 2014 Sep; 130(3):181-92.

56 Klein NS, Kok GD, Burger H, van Valen E, Riper H, Cuijpers $P$, et al. No sustainable effects of an Internet-based relapse prevention program over 24 months in recurrent depression: primary outcomes of a randomized controlled trial. Psychother Psychosom. 2018; 87(1):55-7. 